\title{
Describing the photo-isomerization of a retinal chromophore model with coupled and quantum trajectories
}

\author{
Francesco Talotta, ${ }^{1}$ David Lauvergnat, ${ }^{1}$ and Federica Agostini ${ }^{1}$ \\ ${ }^{1}$ Université Paris-Saclay, CNRS, Institut de Chimie Physique UMR8000, 91405, Orsay, France
}

\begin{abstract}
The exact factorization of the electron-nuclear wavefunction is applied to the study of the photoisomerization of a retinal chromophore model. We describe such an ultrafast nonadiabatic process by analyzing the time-dependent potentials of the theory and by mimicking nuclear dynamics with quantum and coupled trajectories. The time-dependent vector and scalar potentials are the signature of the exact factorization, as they guide nuclear dynamics by encoding the complete electronic dynamics and including excited-state effects. Analysis of the potentials is, thus, essential - when possible - to predict the time-dependent behavior of the system of interest. In this work, we employ the exact time-dependent potentials, available for the numerically-exactly solvable model used here, to propagate quantum nuclear trajectories representing the isomerization reaction of the retinal chromophore. The quantum trajectories are the best possible trajectory-based description of the reaction when using the exact-factorization formalism, and thus allow us to assess the performance of the coupled-trajectory, fully approximate, schemes derived from the exact-factorization equations.
\end{abstract}

\section{INTRODUCTION}

Trajectory-based schemes are widely employed to simulate excited-state nonadiabatic dynamics in molecular systems [1-7]. The main reason for their success is twofold: first, when using trajectories it is possible to perform on-the-fly electronic structure calculations for the visited geometries along the nuclear dynamics [8-10], thus avoiding constructing the electronic potential energy surfaces and related quantities over the whole configuration space [5, 11]; second, evolving trajectories rather than the molecular wavefunction allows one to overcome the exponential scaling of the computational cost with the number of degrees of freedom inherent a fully quantum-mechanical calculation. While the outcome of the dynamics simulation is clearly affected by the accuracy of the underlying electronic-structure method [12], the overall evolution of the system, i.e., coupled nuclei and electrons, strongly depends on the forces used to propagate the trajectories and on the electron-nuclear coupling - intended here as the nuclear feedback on electronic dynamics.

Among the trajectory-based methods derived from the molecular time-dependent Schrödinger equation are, for instance, the direct-dynamics variational multiconfigurational Gaussian (DD-vMCG) [13, 14], ab initio multiple spawning (AIMS) $[3,15,16]$, and ab initio multiple cloning (AIMC) [17, 18]. There, nuclear trajectories are evolved according to variationally-determined (DD-vMCG), or adiabatic (AIMS), or mean-field (AIMC with multiconfigurational Ehrenfest) forces, and are used to guide the basis functions appearing in the expansion of the molecular wavefunction. Electron-nuclear coupling is obtained by solving, alongside the trajectories, the quantum evolution equation for the expansion coefficients. In addition, spawning or cloning procedures aim to improve over time the sampling of the nuclear configuration space. An approximate solution of the molecular time-dependent Schrödinger equation can be obtained via quantum-classical approaches as well, like Ehrenfest dynamics [19, 20] and trajectory surface hopping (TSH) [21]. The former is a mean-field method, where the classical nuclear force and the electron-nuclear coupling are given by averaging either over the electronic or over the nuclear time-dependent states of the system. The latter evolves classical trajectories according to adiabatic forces, and the electronic state of the system according to a Schrödinger equation where the nuclear trajectory appears as a time-dependent parameter. To account for nonadiabatic effects TSH is supplemented with a hopping procedure allowing the trajectories to change electronic state over time.

Trajectory-based schemes [22-27] have also been derived from the exact factorization [28, 29], which is essentially a rewriting of the molecular Schrödinger equation in terms of coupled electronic and nuclear equations. This formalism introduces the new concepts of time-dependent vector potential [30-32] and timedependent scalar potential, or time-dependent potential energy surface [33-35]. They govern the nuclear quantum-mechanical evolution and encode the full dynamical effect of the electrons, including nonadiabatic effects [36-39]. In turn, the electron-nuclear coupling is explicitly given in terms of the spatial variation of the nuclear wavefunction [40, 41]. Evolving classicallike nuclei according to the force determined from the time-dependent potentials and reconstructing the nuclear distribution from an ensemble of trajectories yields the coupled-trajectory mixed quantum-classical (CT-MQC) algorithm [22]. Simplification of this numerical procedure have been proposed, by combining the adiabatic evolution of the trajectories and the hopping idea of TSH with the reconstruction of the nuclear distribution, either by using coupled trajectories, i.e., CT-TSH [25], or by using auxiliary trajectories, i.e., SHXF [27, 42-46].

In this work we aim to assess the performance of coupled-trajectory methods derived from the exact factorization in describing the photo-isomerization reaction 
in a retinal chromophore model [47]. The assessment of CT-MQC and CT-TSH will be carried out based on the benchmark against quantum vibronic wavepacket dynamics - already performed for CT-MQC in previous work [48] - as well as based on the comparison with quantum trajectories $[32,49]$. Since our working framework is the exact factorization, quantum trajectories are determined by evolving them with the (exact) time-dependent potentials of the theory. The potentials are constructed from the solution of the time-dependent Schrödinger equation and, thus, can be viewed as incorporating all quantum dynamical effects of the electrons on the nuclei in nonadiabatic conditions. Our quantum trajectories are, in this sense, the best possible trajectory-based description of the photo-isomerization process, thus allowing us to assess how well the coupled-trajectory approximate methods (could) perform. In addition, we will analyze the time-dependent potentials themselves in order to show that despite the used retinal model is very simple (two-dimensional in nuclear space with two electronic states) their behavior at long times is complex and, consequently, difficult to capture in a trajectory-based description of the dynamics, as was pointed out in previous work.

The paper is organized as follows. In Section II we briefly recall the exact factorization and, in Section II A, the coupled-trajectory algorithms derived from it. Our numerical results are presented in Section III, focusing first on the time-dependent potentials, in Section III A, and then on the analysis of the trajectories, in Section III B. Our conclusions are stated in Section IV.

\section{THEORETICAL FRAMEWORK}

The non-relativistic molecular Hamiltonian

$$
\hat{H}(\mathbf{r}, \mathbf{R})=\sum_{\nu=1}^{N_{n}} \frac{-\hbar^{2} \nabla_{\nu}^{2}}{2 M_{\nu}}+\hat{H}_{e l}(\mathbf{r}, \mathbf{R})
$$

describes a system of interacting electrons and nuclei, whose positions are collectively indicated as $\mathbf{r}$ and $\mathbf{R}$, respectively. The nuclear kinetic energy operator is expressed in Cartesian coordinates and contains a sum over the $N_{n}$ nuclei, each labeled with the index $\nu$, with spatial derivatives $\nabla_{\nu}$ with respect to nuclear positions; $M_{\nu}$ are the nuclear masses. The electronic Hamiltonian $\hat{H}_{e l}(\mathbf{r}, \mathbf{R})$ is the sum of the electronic kinetic energy and of all interactions. The time evolution of the electron-nuclear system is dictated by the timedependent Schrödinger equation (TDSE)

$$
i \hbar \partial_{t} \Psi(\mathbf{r}, \mathbf{R}, t)=\hat{H}(\mathbf{r}, \mathbf{R}) \Psi(\mathbf{r}, \mathbf{R}, t)
$$

whose solution yields the time-dependent molecular wavefunction $\Psi(\mathbf{r}, \mathbf{R}, t)$.

When introducing the exact factorization [4, 28], the molecular wavefunction is written as the product

$$
\Psi(\mathbf{r}, \mathbf{R}, t)=\chi(\mathbf{R}, t) \Phi(\mathbf{r}, t ; \mathbf{R})
$$

where the nuclear wavefunction $\chi(\mathbf{R}, t)$ evolves according to the nuclear TDSE

$$
\begin{aligned}
i \hbar \partial_{t} \chi & (\mathbf{R}, t)= \\
\quad & {\left[\sum_{\nu=1}^{N_{n}} \frac{\left[-i \hbar \nabla_{\nu}+\mathbf{A}_{\nu}(\mathbf{R}, t)\right]^{2}}{2 M_{\nu}}+\epsilon(\mathbf{R}, t)\right] \chi(\mathbf{R}, t) }
\end{aligned}
$$

and the electronic conditional factor $\Phi(\mathbf{r}, t ; \mathbf{R})$, parametrically depending on $\mathbf{R}$, evolves according to the electronic equation

$$
\begin{aligned}
i \hbar \partial_{t} \Phi & (\mathbf{r}, t ; \mathbf{R})= \\
& {\left[\hat{H}_{B O}(\mathbf{r}, \mathbf{R})+\hat{U}_{e n}[\Phi, \chi]-\epsilon(\mathbf{R}, t)\right] \Phi_{\mathbf{R}}(\mathbf{r}, t) }
\end{aligned}
$$

Both evolution equations (4) and (5) contain the time-dependent vector potential (TDVP) $\mathbf{A}_{\nu}(\mathbf{R}, t)$ and the time-dependent potential energy surface (TDPES) $\epsilon(\mathbf{R}, t)$ defined as

$$
\begin{aligned}
& \mathbf{A}_{\nu}(\mathbf{R}, t)=\left\langle\Phi(t ; \mathbf{R}) \mid-i \hbar \nabla_{\nu} \Phi(t ; \mathbf{R})\right\rangle_{\mathbf{r}} \\
& \epsilon(\mathbf{R}, t)=\left\langle\Phi(t ; \mathbf{R})\left|\hat{H}_{B O}(\mathbf{R})+\hat{U}_{e n}[\Phi, \chi]-i \hbar \partial_{t}\right| \Phi(t ; \mathbf{R})\right\rangle_{\mathbf{r}}
\end{aligned}
$$

The symbol $\langle\cdot\rangle_{\mathbf{r}}$ stands for an integration over the electronic degrees of freedom, and we removed all dependencies on $\mathbf{r}$ within $\langle\cdot\rangle_{\mathbf{r}}$ to imply that this variable is integrated out. The TDVP and the TDPES are electronic quantities that evolve in time as effect of electronic dynamics, which is coupled to the nuclear dynamics in nonadiabatic conditions, and completely determine the nuclear evolution via the nuclear TDSE (4). It is interesting to note that the TDVP is related to the nuclear momentum field, as

$$
\mathbf{A}_{\nu}(\mathbf{R}, t)=\frac{\operatorname{Im}\left[\left\langle\Psi(\mathbf{R}, t)\left|\hbar \nabla_{\nu}\right| \Psi(\mathbf{R}, t)\right\rangle_{\mathbf{r}}\right.}{|\chi(\mathbf{R}, t)|^{2}}-\nabla_{\nu} S(\mathbf{R}, t)
$$

a property that will be used in Section II A to define our "quantum trajectories". The symbol $S(\mathbf{R}, t)$ is used to indicate the phase of the nuclear wavefunction $\chi(\mathbf{R}, t)$ and it has the dimensions of an action. The electronic equation (5) contains the electron-nuclear coupling operator

$$
\begin{aligned}
& \hat{U}_{e n}[\Phi, \chi]=\sum_{\nu=1}^{N_{n}} \frac{1}{M_{\nu}}\left[\frac{\left[-i \hbar \nabla_{\nu}-\mathbf{A}_{\nu}(\mathbf{R}, t)\right]^{2}}{2}\right. \\
& \left.\quad+\left(\frac{-i \hbar \nabla_{\nu} \chi(\mathbf{R}, t)}{\chi(\mathbf{R}, t)}+\mathbf{A}_{\nu}(\mathbf{R}, t)\right)\left(-i \hbar \nabla_{\nu}-\mathbf{A}_{\nu}(\mathbf{R}, t)\right)\right]
\end{aligned}
$$

which depends explicitly on the nuclear wavefunction, on the electronic wavefunction via the presence of the TDVP, and acts on the parametric dependence of $\Phi(\mathbf{r}, t ; \mathbf{R})$ as a spatial derivative.

The ambiguity of the product form of the molecular wavefunction in Eq. (3) is partially eliminated by imposing the normalization condition $\int|\Phi(\mathbf{r}, t ; \mathbf{R})|^{2} d \mathbf{r}=$ 
$1 \forall \mathbf{R}, t$, which allows to identify $|\Phi(\mathbf{r}, t ; \mathbf{R})|^{2}$ as a conditional probability density and $|\chi(\mathbf{R}, t)|^{2}=$ $\int|\Psi(\mathbf{r}, \mathbf{R}, t)|^{2} d \mathbf{r}$ as a marginal probability density that yields the nuclear density from the full wavefunction. Equation (3) is thus unique up to a gauge encoded in a phase factor $e^{(i / \hbar) \theta(\mathbf{R}, t)}$ (with $\theta(\mathbf{R}, t)$ a real function): multiplying the nuclear wavefunction by this factor and the electronic term by its complex conjugate, Eq. (3) remains unaffected and the time-dependent potentials transform as standard gauge potentials. Therefore, this ambiguity has to be eliminated by imposing a choice of gauge as discussed in Section II A.

\section{A. Trajectory-based description}

In a trajectory-based description of the coupled electron-nuclear dynamics in the presence of nonadiabatic effects and described within the exact-factorization framework, nuclear dynamics from Eq. (4) is approximated in terms of an ensemble of coupled trajectories. Their positions and momenta evolve according to Hamilton equations under the effect of the TDVP and TDPES [50]. In turn, the TDVP and TDPES are computed from the electronic wavefunction, which is expanded in the adiabatic basis, i.e., the basis of eigenstates of $\hat{H}_{e l}(\mathbf{r}, \mathbf{R})$. Equation (5) yields evolution equations for the expansion coefficients, which are solved coupled to the nuclear Hamilton equations. This is the essence of the coupled-trajectory mixed quantum-classical (CTMQC) algorithm [22]. It has been abundantly described in the literature, therefore, we refer the interested reader to Refs. [4, 51-53] for in-depth discussions, and we recall here only the main ideas and equations.

A nuclear trajectory, indicated with the symbol $\mathbf{R}^{\alpha}(t)$, is intended as a collection of $3 N_{n}$ positions that evolve in time. When the electronic wavefunction $\Phi\left(\mathbf{r}, t ; \mathbf{R}^{\alpha}(t)\right)$ is expressed as a linear combination of adiabatic states, $\varphi^{(m)}\left(\mathbf{r} ; \mathbf{R}^{\alpha}(t)\right)$, their parametric dependence on the nuclear position/trajectory induces an implicit time dependence. The expansion coefficients in $\Phi\left(\mathbf{r}, t ; \mathbf{R}^{\alpha}(t)\right)=$ $\sum_{m} C_{m}\left(\mathbf{R}^{\alpha}(t), t\right) \varphi^{(m)}\left(\mathbf{r} ; \mathbf{R}^{\alpha}(t)\right)$ depend on the nuclear trajectory as well. In the following, the dependence on $\mathbf{R}^{\alpha}(t)$ will be indicated only via the index $\alpha$ for simplicity.

For the trajectory $\alpha$, the classical force on the nucleus $\nu$ at time $t$ is $\mathbf{F}_{\nu}^{\alpha}(t)$ and is written as the sum of three terms

$$
\mathbf{F}_{\nu}^{\alpha}(t)=\mathbf{F}_{\nu, \mathrm{mf}}^{\alpha}(t)+\mathbf{F}_{\nu, \text { na }}^{\alpha}(t)+\mathbf{F}_{\nu, \mathrm{ct}}^{\alpha}(t)
$$

The mean-field contribution $\mathbf{F}_{\nu, \mathrm{mf}}^{\alpha}(t)$ is the average of adiabatic forces $-\nabla_{\nu} E_{m}^{\alpha}$ over the electronic states weighted by the population of the states $\left|C_{m}^{\alpha}(t)\right|^{2}$, namely

$$
\mathbf{F}_{\nu, \mathrm{mf}}^{\alpha}(t)=\sum_{m}\left|C_{m}^{\alpha}(t)\right|^{2}\left(-\nabla_{\nu} E_{m}^{\alpha}\right)
$$

The adiabatic energies are indicated as $E_{m}$. The nonadiabatic contribution $\mathbf{F}_{\nu, \text { na }}^{\alpha}(t)$ depends explic- itly on the nonadiabatic coupling vectors $\mathbf{d}_{\nu, m l}^{\alpha}=$ $\left\langle\varphi^{(m)}\left(\mathbf{R}^{\alpha}(t)\right) \mid \nabla_{\nu} \varphi^{(l)}\left(\mathbf{R}^{\alpha}(t)\right)\right\rangle_{\mathbf{r}}$ and is

$$
\mathbf{F}_{\nu, \mathrm{na}}^{\alpha}(t)=\sum_{m, l} \bar{C}_{m}^{\alpha}(t) C_{l}^{\alpha}(t)\left(E_{m}^{\alpha}-E_{l}^{\alpha}\right) \mathbf{d}_{\nu, m l}^{\alpha}
$$

with $\bar{C}_{m}^{\alpha}(t)$ the complex conjugate of $C_{m}^{\alpha}(t)$. The last contribution $\mathbf{F}_{\nu, \mathrm{ct}}^{\alpha}(t)$ arises as effect of the coupling among the trajectories

$\mathbf{F}_{\nu, \mathrm{ct}}^{\alpha}(t)=\frac{2}{\hbar} \sum_{m}\left|C_{m}^{\alpha}(t)\right|^{2}\left[\sum_{\mu=1}^{N_{n}} \mathcal{P}_{\mu}^{\alpha}(t) \cdot \mathbf{f}_{\mu, m}^{\alpha}\right]\left(\mathbf{f}_{\nu, m}^{\alpha}-\mathbf{A}_{\nu}^{\alpha}(t)\right)$

and depends on the TDVP $\mathbf{A}_{\nu}^{\alpha}(t)$, on the, so-called, accumulated adiabatic force $\mathbf{f}_{\nu, m}^{\alpha}=\int_{0}^{t}\left(-\nabla_{\nu} E_{m}^{\alpha}\right) d \tau$, and on the quantum momentum $\mathcal{P}_{\mu}^{\alpha}(t)$. Calculation of the quantum momentum requires knowledge of the positions of all trajectories at a given time $t$, thus the trajectories cannot be evolved independently [54]. The quantum momentum follows from the term in the expression of the electron-nuclear coupling operator (9) that depends explicitly on the nuclear wavefunction; its expression is related to the spatial variation of the nuclear density as

$$
\mathcal{P}_{\mu}^{\alpha}(t)=\frac{-\hbar \nabla_{\mu}\left|\chi\left(\mathbf{R}^{\alpha}(t), t\right)\right|^{2}}{2\left|\chi\left(\mathbf{R}^{\alpha}(t), t\right)\right|^{2}}
$$

In CT-MQC the nuclear density is reconstructed as a sum of frozen Gaussians centered at the positions of the trajectories, and the quantum momentum is determined from Eq. (14).

The electronic equation (5) yields evolution equations for the expansion coefficients $C_{m}^{\alpha}(t)$ in the form

$$
\dot{C}_{m}^{\alpha}(t)=\dot{C}_{m, \mathrm{TSH}}^{\alpha}(t)+\dot{C}_{m, \mathrm{ct}}^{\alpha}(t)
$$

where the first term is identical as in a standard trajectory surface hopping (TSH) procedure [21]

$$
\dot{C}_{m, \mathrm{TSH}}^{\alpha}(t)=-\frac{i}{\hbar} E_{m}^{\alpha} C_{m}^{\alpha}(t)-\sum_{l} \sum_{\nu=1}^{N_{n}} \dot{\mathbf{R}}_{\nu}^{\alpha}(t) \cdot \mathbf{d}_{\nu, m l}^{\alpha} C_{l}^{\alpha}(t)
$$

with $\dot{\mathbf{R}}_{\nu}^{\alpha}(t)$ the nuclear velocity, and the additional term $\dot{C}_{m, \mathrm{ct}}^{\alpha}(t)$ depends on the quantum momentum

$$
\dot{C}_{m, \mathrm{ct}}^{\alpha}(t)=\sum_{\nu=1}^{N_{n}} \frac{\mathcal{P}_{\nu}^{\alpha}(t)}{\hbar M_{\nu}} \cdot\left(\mathbf{f}_{\nu, m}^{\alpha}-\mathbf{A}_{\nu}^{\alpha}(t)\right) C_{m}^{\alpha}(t)
$$

similarly to the expression of the force.

In deriving CT-MQC equations, the gauge has been chosen such that $\epsilon^{\alpha}(t)+\sum_{\nu} \dot{\mathbf{R}}_{\nu}^{\alpha}(t) \cdot \mathbf{A}_{\nu}^{\alpha}(t)=0$, with $\epsilon^{\alpha}(t)$ the value of the TDPES at the position of the trajectory.

Recently, the coupled-trajectory idea of CT-MQC, encoded in the quantum momentum, was combined with a TSH procedure to derive the CT-TSH scheme. CT-TSH 
dynamics promises to be more stable than CT-MQC at long times, at least when, as it was shown for a onedimensional system [25], the TDPES develops an oscillatory behavior. This can happen if the trajectories cross a nonadiabatic region several times and interfere. In addition, the computational cost for the force calculation is reduced if compared to CT-MQC because the nonadiabatic force, depending on the nonadiabatic coupling vectors, does not appear. In CT-TSH, the force driving a trajectory is simply determined from the gradient of the adiabatic energy of the running state, i.e., the state associated to that trajectory at a given time. The running state changes over time as a trajectory can hop from one state to another according to the standard fewest switches procedure. The probability for a hop from state $m$ to state $l$ to occur at time $t$ is determined as [9]

$$
P_{m l}^{\alpha}(t)=\max \left[0, \frac{-2 \bar{C}_{m}^{\alpha}(t) C_{l}^{\alpha}(t) \sum_{\nu} \mathbf{d}_{\nu, l m}^{\alpha} \cdot \dot{\mathbf{R}}_{\nu}^{\alpha}(t)}{\left|C_{m}^{\alpha}(t)\right|^{2}} d t\right]
$$

with $d t$ the integration time step. Being $P_{m l}^{\alpha}(t)$ a real quantity, one has to take the real part of the numerator in brackets. CT-TSH trajectories remain, however, coupled, because Eq. (15) is used to propagate the electronic coefficients, as in CT-MQC, thus the quantum momentum has to be computed. When the electronic evolution equation of standard TSH is complemented with the coupled-trajectory term of Eq. (17) the overcoherence problem of TSH is overcome, since the quantum momentum allows one to account for decoherence effects, as shown in previous work $[22,24,25,54]$.

In CT-MQC and CT-TSH both the electronic and the nuclear equations of the exact factorization are approximated, as described above. Therefore, a question that is indeed interesting to address is: How well can one hope to reproduce nuclear dynamics using trajectories? To answer this question, we introduce the concept of quantum trajectories, as trajectories that are propagated under the effect of the exact TDVP and TDPES [32]. For a model system, the TDVP and the TDPES can be computed numerically exactly from the output of vibronic wavepacket dynamics. Furthermore, if a gauge is chosen such that the TDVP is the nuclear momentum field [30-32], then the simple equation

$$
\dot{\mathbf{R}}_{\nu}^{\alpha}(t)=\frac{\mathbf{A}_{\nu}^{\alpha}(t)}{M_{\nu}}
$$

can be integrated to determine the positions of the trajectories at all times that closely follow the evolution of the (exact) nuclear density. Here, $\mathbf{A}_{\nu}^{\alpha}(t)$ stands for the value of the exact TDVP at the position $\mathbf{R}_{\nu}^{\alpha}(t)$. In the gauge where the nuclear wavefunction is always real and non-negative, the second term on the right-hand side of Eq. (8) identically vanishes and the TDVP equals the nuclear momentum field calculated from the molecular wavefunction. Therefore, to determine the (numerically exact) TDVP, the solution of the TDSE (2) is integrated over electronic positions to get the nuclear density, whose positive square root gives the nuclear wavefunction in this gauge. Dividing the molecular wavefunction by the nuclear wavefunction yields the electronic wavefunction, from Eq. (3), and Eq. (6) can be directly used to determine the TDVP.

Note that we refer to the trajectories determined according to Eq. (19) as quantum trajectories in a (Bohmian) hydrodynamic sense. If one focuses only on the nuclear TDSE (4), then the TDVP is the Bohmian definition of the momentum field for quantummechanical particles described by a real wavefunction in the presence of an external vector potential [55]. It was recently proven [32] that this procedure is equivalent to propagating trajectories according to forces that include the combined effect of the TDPES and of the quantum potential - usually arising from the Bohmian formulation of quantum dynamics. In addition, the analysis reported here differs from the setup of Ref. [37] where the dynamics of the trajectories was driven only by the TDPES (without the quantum potential) and, thus, the trajectories were purely "classical".

\section{NUMERICAL RESULTS}

The model Hamiltonian [47] used in this work allows us to describe the 11-cis to all-trans isomerization of the retinal chromophore in rhodopsin [30, 56-67]. Photoabsorption takes the cis conformer from the electronic ground state $S_{0}$ to the first excited state $S_{1}$, thus initiating an isomerization reaction towards the trans conformer. The nuclear coordinates defining the model are an angular reaction coordinate $\varphi$ and a collective vibration $q$ characterizing "a delocalized stretching motion of the polyene chain, whereby single and double bonds interchange" [47].

The Hamiltonian is given in the diabatic basis

$$
\hat{H}(\varphi, q)=\frac{\hat{P}_{\varphi}^{2}}{2 m}+\frac{\hat{P}_{q}^{2}}{2 \omega^{-1}}+\left(\begin{array}{cc}
V_{00}(\varphi, q) & V_{01}(q) \\
V_{10}(q) & V_{11}(\varphi, q)
\end{array}\right)
$$

supposing here that $\mathbf{R}=\varphi, q$ (dimensionless coordinates). The nuclear kinetic energy operator is expressed via the momentum operators along $\varphi$ and $q$, where $\hat{P}_{\varphi}^{2}$ and $\hat{P}_{q}^{2}$ are second-order derivatives with respect to $\varphi$ and $q$, respectively. The elements of the $2 \times 2$ electronic matrix are

$$
\begin{aligned}
& V_{00}(\varphi, q)=\frac{1}{2} W_{0}(1-\cos \varphi)+\frac{1}{2} \omega q^{2} \\
& V_{11}(\varphi, q)=E_{1}-\frac{1}{2} W_{1}(1-\cos \varphi)+\frac{1}{2} \omega q^{2}+\kappa q \\
& V_{01}(q)=V_{10}(q)=\lambda q
\end{aligned}
$$

The stretching mode $q$ is also called coupling mode, as the potential coupling $V_{10}$ only depends on $q$. The effective mass of the reaction coordinate $m^{-1}=4.84 \cdot 10^{-4} \mathrm{eV}$ 
is chosen so as to achieve isomerization within $200 \mathrm{fs}$; the frequency of the coupling mode is $\omega=0.19 \mathrm{eV}$ and the interstate coupling is $\lambda=0.19 \mathrm{eV}$, with $\kappa=0.1 \mathrm{eV}$ indicating a gradient in the excited state; the parameters $W_{0}=3.6 \mathrm{eV}, W_{1}=1.09 \mathrm{eV}$ and $E_{1}=2.48 \mathrm{eV}$ are chosen in order to match the $\mathrm{S}_{0} / \mathrm{S}_{1}$ gap to the center frequency of the absorption bands of the cis and trans isomers. The adiabatic potential energy surfaces (PESs) $\mathrm{S}_{0}$ and $\mathrm{S}_{1}$ are obtained by diagonalization of the electronic Hamiltonian in Eq. (20). The PESs present conical intersections (CIs) at $\varphi_{C I} \simeq \pm \frac{\pi}{2}, q_{C I}=0$. The cis conformer is characterized by the value of the reactive coordinate $\varphi_{\text {cis }}=0$, and at this geometry for $q=0$ the excitation energy from $\mathrm{S}_{0}$ to $\mathrm{S}_{1}$ lies in the visible domain. The adiabatic PESs are shown in Fig. 1 as colormaps, where the positions of the CIs are indicated with crosses. At the cis geometry and for slightly negative values of the coupling mode, $\mathrm{S}_{0}$ is a potential well while $S_{1}$ has a saddle shape; at the trans geometry $(\varphi= \pm \pi)$, only $\mathrm{S}_{0}$ presents stable configurations.

The instantaneous excitation from $\mathrm{S}_{0}$ to $\mathrm{S}_{1}$ promotes the ground-state wavepacket to the excited state. The ground-state wavepacket is chosen as a two-dimensional Gaussian

$$
\chi_{\mathrm{S}_{0}}(\varphi, q, 0)=\sqrt[4]{\frac{1}{\pi \sigma_{\varphi}^{2}}} e^{-\frac{\varphi^{2}}{2 \sigma_{\varphi}^{2}}} \sqrt[4]{\frac{1}{\pi \sigma_{q}^{2}}} e^{-\frac{q^{2}}{2 \sigma_{q}^{2}}}
$$

with $\sigma_{\varphi}=0.128$ and $\sigma_{q}=1.0$, which corresponds to the vibrational ground state of $\mathrm{S}_{0}$ at the cis geometry. The nuclear (probability) density at all times is given as the sum of the $\mathrm{S}_{0}$ and $\mathrm{S}_{1}$ (probability) densities

$$
|\chi(\varphi, q, t)|^{2}=\left|\chi_{\mathrm{S}_{0}}(\varphi, q, t)\right|^{2}+\left|\chi_{\mathrm{S}_{1}}(\varphi, q, t)\right|^{2}
$$

Electronic populations of the ground $\left(\mathrm{S}_{0}\right)$ or excited $\left(\mathrm{S}_{1}\right)$ states are determined as the integral of the corresponding nuclear density,

$$
\rho_{\mathrm{S}_{k}}(t)=\iint\left|\chi_{\mathrm{S}_{k}}(\varphi, q, t)\right|^{2} d \varphi d q \quad \text { with } k=0,1
$$

Vibronic wavepacket dynamics after photo-excitation is simulated in the diabatic basis using ElVibRot [68]. The diabatic densities are transformed to the adiabatic representation for the comparison with the trajectorybased approaches. The nuclear wavepacket has been expanded in a two-dimensional basis set formed by the direct product of two uni-dimensional basis sets for each diabatic state. The harmonic-oscillator basis set has been used for $q$ (40 basis functions), and a Fourier-series, periodic basis set has been used for $\varphi$ (512 basis functions); the number of grid points along each coordinate is 80 and 552, respectively for $q$ and $\varphi$. The propagation was performed with the Chebychev scheme for which the evolution operator is expanded on Chebychev polynomials [69] with time step 0.1 fs. The Hamiltonian needs to be renormalized so that its spectral range lies in the interval $[-1,1]$.

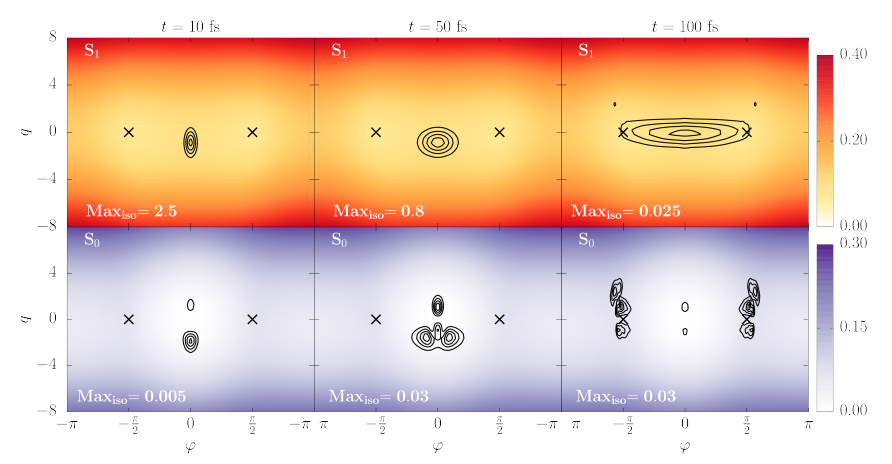

FIG. 1. Quantum nuclear dynamics in terms of adiabatic wavepackets contributions (contour lines) superimposed to the adiabatic PESs (colormaps). In each panel the value

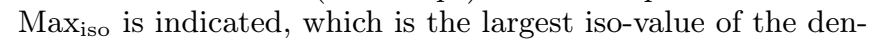
sity represented in the figure. The upper panels refer to $S_{1}$ and the lower panels to $\mathrm{S}_{0}$. The color bars report the energies in Ha. The nuclear densities in $\mathrm{S}_{0}$ and in $\mathrm{S}_{1}$ are shown at times $t=10,50,100 \mathrm{fs}$ as indicated in the figure. The CIs are indicated by the crosses.

Figure 1 reports snapshots of the nuclear dynamics at times $t=10,50,100 \mathrm{fs}$, where the contour lines represent the adiabatic components of the nuclear density (in $\mathrm{S}_{1}$ in the upper panels and in $\mathrm{S}_{0}$ in the lower panels) and the colormaps represent the adiabatic PESs $\left(\mathrm{S}_{1}\right.$ in the upper panels and $S_{0}$ in the lower panels). The initially excited density on $\mathrm{S}_{1}(t=10 \mathrm{fs})$ extends over time towards the CIs $(t=50 \mathrm{fs})$ and finally funnels via the CIs to $\mathrm{S}_{0}$ $(t=100 \mathrm{fs})$. While the dynamics shows coherent oscillations along $q$, the isomerization reaction from the cis to the trans conformer is characterized by the wavepacket reaching the values $|\varphi|>\frac{\pi}{2}$.

The isomerization process just described will be analyzed in detail employing the exact factorization, in Section III A by looking at TDPES and TDVP, and in Section III B by using quantum and coupled trajectories.

\section{A. Time-dependent potentials}

The time-dependent potentials emerging from the exact factorization can be constructed from the output of a vibronic wavepacket quantum simulation. If the electron-nuclear state of the system $|\Psi(\varphi, q, t)\rangle$ is expanded in the diabatic basis with coefficients $\chi_{k}^{(d)}(\varphi, q, t)$ $(k=0,1)$, the nuclear wavefunction in the chosen gauge is $\chi(\varphi, q, t)=\sqrt{\left|\chi_{0}^{(d)}(\varphi, q, t)\right|^{2}+\left|\chi_{1}^{(d)}(\varphi, q, t)\right|^{2}}$. Therefore, when the electronic conditional state $|\Phi(t ; \varphi, q)\rangle$ is expanded in the same basis, the expansion coefficients are $\chi_{k}^{(d)}(\varphi, q, t) / \chi(\varphi, q, t)$ by virtue of the exact factorization (3). Using these coefficients and replacing the integration over $\mathbf{r}$ of Eqs. (6) and (7) with a sum over the diabatic states yields the $(\varphi, q, t)$-dependent TDPES and TDVP. Studying the evolution of their behavior in time allows one to qualitatively interpret and justify the 


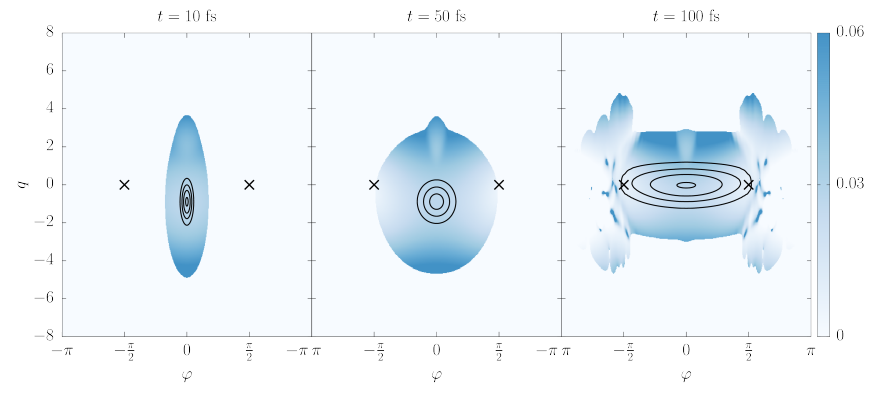

FIG. 2. TDPES (colormaps) at times $t=10,50,100 \mathrm{fs}$ as indicated in the figure. The color bar reports the energy in Ha. The nuclear density (contour lines) at the same times is superimposed for reference. The CIs are indicated by the crosses.

dynamics of the nuclear wavefunction. To compute the TDPES and the TDVP we used the Exact Factorization Analysis Code (EFAC) [70].

\section{Time-dependent potential energy surface}

In Fig. 2 we report the TDPES (colormap) as function of the nuclear coordinates $\varphi$ and $q$ at the same time steps as in Fig. 1, superimposed with the nuclear density $|\chi(\varphi, q, t)|^{2}$ (contour lines) and indicating the CIs as crosses. Note that the TDPES is only calculated, and shown, in regions where the nuclear density is larger than $10^{-7}$ for numerical convenience.

At the beginning of the simulated dynamics, $t=10 \mathrm{fs}$ in Fig. 2 (left panel), the TDPES along $q$ is basically a potential well with minimum between $q=-2$ and $q=0$ where the nuclear density is localized; along $\varphi$ and in the region $q \in[-2,0]$ the TDPES is slightly more flat than along $q$, so as to allow the density to spread towards the CIs. This shape resembles the adiabatic $\mathrm{S}_{1}$ PES, since at this early time, nuclear dynamics fully takes place in the excited state.

At later times, $t=50$ fs in Fig. 2 (central panel), the nuclear density starts spreading symmetrically towards the CIs as consequence of the appearance of a saddle shape along $\varphi$ with maximum in $\varphi=0$ in the region $q \in[-3,0]$. In the regions $q<-3$ and $q>0$, the increase of the TDPES localizes the nuclear density along the $q$ direction. In particular, the shape of the TDPES around $\varphi=0, q=2$ indicates the presence of a small portion of the nuclear density in the ground state. We recall that quantum dynamics is performed in the diabatic basis and, thus, the nuclear state is initialized with full population in the diabatic state $k=1$. This means that a very small amount of density is found in the (adiabatic) ground state even at time $t=0$, whose evolution is also dictated by the TDPES. In the portion of configuration space $q>0$ at $t=50$ fs the (adiabatic) excited-state density is much smaller than the ground-state density, thus the TDPES follows the shape of the $\mathrm{S}_{0}$ PES.
At the final time shown in Fig. 2, $t=100$ fs (right panel), the central portion of the TDPES where the nuclear density is mainly located has, once again, a saddle shape along $\varphi$ with maximum in $\varphi=0$, allowing in this way the nuclear density to reach the CIs and to "funnel" to the ground state, thus accessing the trans configuration. However, note that in a description based on the TDPES, we do not need to invoke any funnelling process to justify the transition from the cis to the trans region, which is, in fact, simply accompanied by the shape of the TDPES. The highly oscillatory features that develop in the colored areas of the TDPES arise from the interferences between the $S_{1}$ density transferred in $S_{0}$ and the $\mathrm{S}_{0}$ density. After $t=100 \mathrm{fs}$ and up to $t=200 \mathrm{fs}$, the shape of the TDPES in the trans region is mainly characterized by interferences between the $\mathrm{S}_{1}$ portion of the density that continuously funnels through the CIs and the $S_{0}$ portion that accumulates there.

It is worth noting that periodic boundary conditions are used along the reactive coordinate $\varphi$. Therefore, interferences are produced as well by $\mathrm{S}_{0}$ portions of the nuclear wavepacket that reach the trans configuration at $+\pi(-\pi)$ and continue their evolution "reentering" the simulation box from $-\pi(+\pi)$.

\section{Time-dependent vector potential}

In Fig. 3 the TDVP offers a complementary perspective on the nuclear dynamics to the TDPES, and, in the used gauge, it is identified with the nuclear momentum field. As for the TDPES, the TDVP is only shown in regions where the nuclear density is larger than $10^{-7}$.

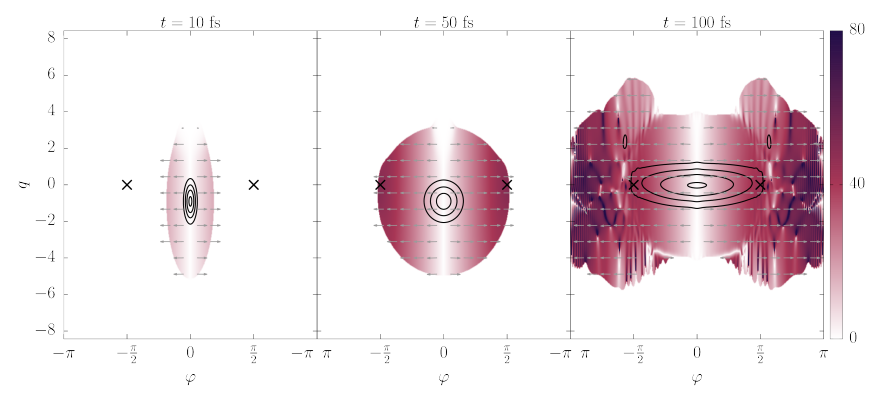

FIG. 3. Magnitude of the TDVP (colormaps) and its direction (unit vector) at times $t=10,50,100 \mathrm{fs}$ as indicated in the figure. The nuclear density (contour lines) at the same times is superimposed for reference. The CIs are indicated by the crosses.

At $t=10 \mathrm{fs}$ (left panel) the TDVP is small in magnitude because the dynamics starts with zero average momentum, and appears symmetric in the $\varphi$ direction with respect to $\varphi=0$. This feature is maintained all along the simulated dynamics, as the nuclear density moves symmetrically towards the CIs. The TDVP reported in all panels in Fig. 3 changes sign in the $\varphi$ direction at $\varphi=0$.

As the nuclear density approaches the CIs, at $t=50 \mathrm{fs}$ 
(central panel), the magnitude of the TDVP increases. This behavior can be easily explained having in mind the double-cone shape of the adiabatic PESs, with the slope of the $\mathrm{S}_{1}$ PES inducing the nuclear density to efficiently relax towards $\mathrm{S}_{0}$ via the CIs.

At the final time shown in Fig. 3, $t=100$ fs (right panel), the magnitude of the TDVP reflects the interference patterns already observed in the TDPES, with a clear separation between the smooth behavior in the cis region $|\varphi|<\frac{\pi}{2}$ and a highly oscillatory behavior mainly identified in the trans region $|\varphi|>\frac{\pi}{2}$.

In all panels in Fig. 3, the vector field representing the direction of the TDVP is nearly parallel to the horizontal axis, indicating that the $q$ component of the momentum field is much smaller than the $\varphi$ component. This results in dynamics mainly characterized by motion from the cis to the trans configuration.

\section{B. Quantum and coupled trajectories}

Quantum trajectories are determined by intergrating Eq. (19) where the TDVP is calculated as described in Section III A from the output of our quantum-dynamics simulation. We refer to $A_{\varphi}(\varphi, q, t)$ and to $A_{q}(\varphi, q, t)$ as the two components of the TDVP. Initial conditions for the quantum trajectories are determined by sampling 1000 positions $\mathbf{R}^{\alpha}(0)=\varphi^{\alpha}(0), q^{\alpha}(0)$ from the Gaussian probability distribution $\left|\chi_{\mathrm{S}_{0}}(\varphi, q, 0)\right|^{2}$ associated to the chosen initial condition for the quantum simulation. Once the initial positions are given, the initial velocities are simply determined as $\dot{\varphi}^{\alpha}(0), \dot{q}^{\alpha}(0)=$ $A_{\varphi}\left(\varphi^{\alpha}(0), q^{\alpha}(0), 0\right) / m, A_{q}\left(\varphi^{\alpha}(0), q^{\alpha}(0), 0\right) / \omega^{-1}$.

Coupled trajectories are propagated according to the CT-MQC and CT-TSH algorithms. We used 1000 initial conditions, whose positions and momenta are independently sampled from the Wigner distribution determined from $\left|\chi_{\mathrm{S}_{0}}(\varphi, q, 0)\right|^{2}$ (the initial positions are identical to the initial quantum trajectories). In the case of CTMQC, we extract nuclear and electronic observables by averaging over 1000 trajectories. In the case of CT-TSH, we perform 4 independent runs in order to account for different histories of hops, thus yielding a total of 4000 trajectories whose results are averaged to extract the observables that will be discussed below.

CT-MQC and CT-TSH dynamics are performed in the adiabatic basis, with adiabatic PESs determined by diagonalizing the electronic Hamiltonian in Eq. (20). The nonadiabatic couplings are computed analytically with the usual Hellmann-Feynman theorem [71] from the eigenvectors of the electronic Hamiltonian in Eq. (20). This procedure is implemented in the ModelLib library [72]. The velocity-Verlet algorithm is used to integrate classical nuclear equations, and the fourth-order Runge-Kutta method is used for the electronic evolution; nuclear and electronic dynamics are integrated with at time step of $0.0024 \mathrm{fs}(0.1 \mathrm{au})$. We checked the stability of CT-TSH results by reducing/increasing by half the

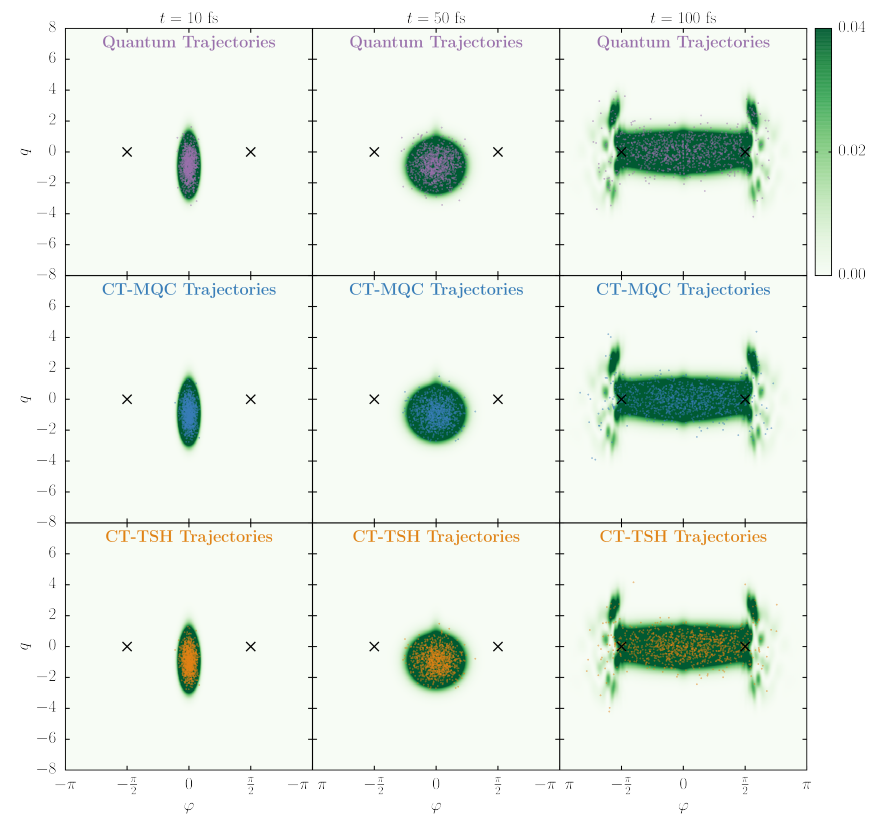

FIG. 4. Exact nuclear density (colormap) at times $t=$ $10,50,100 \mathrm{fs}$ as indicated in the figure, compared with the positions of the quantum trajectories (lilac dots in the upper panel), CT-MQC trajectories (blue dots in the middle panels) and CT-SH trajectories (orange dots in the lower panels) at the same times. The CIs are indicated by the crosses.

integration time step, since the hopping probability of Eq. (18) depends on it. The electronic initial conditions are chosen as $C_{\mathrm{S}_{0}}^{\alpha}(0)=(0.0,0.0), C_{\mathrm{S}_{1}}^{\alpha}(0)=(1.0,0.0) \forall \alpha$. Calculations based on coupled trajectories have been performed with G-CTMQC [73].

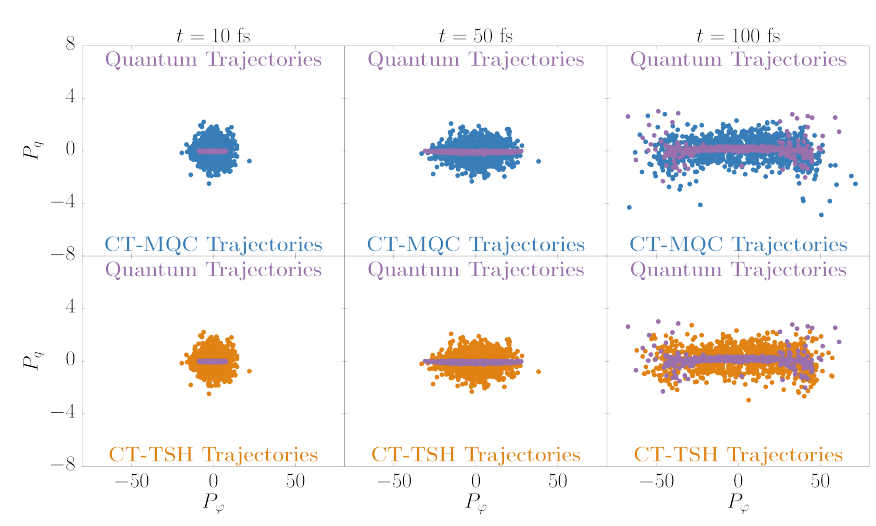

FIG. 5. Distribution of the trajectories in momentum space at times $t=10,50,100 \mathrm{fs}$ as indicated in the figure. Upper panels: Comparison between quantum trajectories (lilac dots) and CT-MQC trajectories (blue dots). Lower panels: Comparison between quantum trajectories (lilac dots) and CTTSH trajectories (orange dots). 


\section{Distributions of trajectories}

In Fig. 4 we compare the distributions of nuclear trajectories with the quantum nuclear density (green areas) at times $t=10,50,100 \mathrm{fs}$ along the dynamics. Quantum trajectories are indicated as lilac dots in the upper panels, CT-MQC trajectories as blue dots in the middle panels and CT-TSH trajectories as orange dots in the lower panels. In general, we observe that CT-MQC and CT-TSH trajectories resemble closely the distribution of quantum trajectories, in agreement with the initialization of all trajectories ensembles from the same distribution in configuration space.

In Fig. 5 the distributions of momenta are shown at times $t=10,50,100 \mathrm{fs}$ along the dynamics. In the upper panels, CT-MQC distributions (blue dots) are compared to the quantum trajectories (lilac dots), whereas, in the lower panels, CT-TSH distributions (orange dots) are compared to the quantum trajectories. In both cases a clear qualitative difference is observed, which follows from the different initial momenta used for the quantum and for the coupled trajectories. In CT-MQC and CTTSH positions and momenta are independent variables, and thus they are randomly sampled from uncorrelated (Wigner-transformed) positions-momenta distributions. Note that the distributions are uncorrelated since the (quantum) initial density is a two-dimensional Gaussian in the variables $\varphi$ and $q$. Figure 5 shows that the momenta distribution of quantum trajectories is much more localized along $P_{q}$ than the distributions of coupled trajectories, whereas they have comparable widths along $P_{\varphi}$ at time $t=10 \mathrm{fs}$ (left panels). The momenta of the quantum trajectories rapidly increase only along $P_{\varphi}$, and the distribution in this direction at time $t=50 \mathrm{fs}$ (central panels) is as broad as CT-MQC and CT-TSH. At later times, $t=100 \mathrm{fs}$ in the figure (right panel), the distributions of all ensembles of trajectories have comparable broadening along $P_{\varphi}$. However, quantum trajectories remain extremely localized in momentum space along $P_{q}$ if compared to CT-MQC and CT-TSH.

The results shown in Fig. 5 are consistent with the previously observed property of the TDVP of being much larger along the $\varphi$ direction than along the $q$ direction. In Fig. 3 the vector field representing the direction of the TDVP is basically parallel to the horizontal axis. Fig. 5 shows, in fact, that $P_{\varphi}$ attains values up to approximately 50 times the values of $P_{q}$.

In general, Figs. 4 and 5 show that the initial dynamics simulated with CT-MQC and CT-TSH is basically identical, and this is because the trajectories evolve adiabatically on $\mathrm{S}_{1}$. When they reach the CIs, however, the treatment of the dynamics differs regarding both the nuclear forces and the evolution of the electronic coefficients. When and after the nonadiabatic event occurs, CT-MQC and CT-TSH yield (slightly) different results. The analysis of Figs. 4 and 5 also suggests that we can directly compare average configurational properties extracted from quantum trajectories and from CT-MQC/CT-TSH tra- jectories. On the other hand, the behavior of quantum and coupled (or classical-like) trajectories in momentum space is qualitatively different as consequence of the different relations between initial positions and initial momenta in the two schemes.

\section{Averaged observables}

Figure 6 shows the population of the electronic excited state $S_{1}$ as function of time (left panel), where reference results are provided by Eq. (26) and are indicated in red. For CT-MQC (blue) and CT-TSH (orange) this same observable is estimated as the average over the trajectories of $\left|C_{\mathrm{S}_{1}}^{\alpha}(t)\right|^{2}$, namely as

$$
\rho_{\mathrm{S}_{1}}^{\mathrm{q} \cdot}(t)=\frac{1}{N_{\text {traj }}} \sum_{\alpha=1}^{N_{\text {traj }}}\left|C_{\mathrm{S}_{1}}^{\alpha}(t)\right|^{2}
$$

We refer to this average as "quantum" and we indicate it as (q.) in Fig. 6. In addition, in CT-TSH, one can also count how many trajectories are "on" the $\mathrm{S}_{1}$ PES at time $t$, i.e., $N_{\mathrm{S}_{1}}(t)$, and divide this number by the total number of trajectories (green line in Fig. 6), namely

$$
\rho_{\mathrm{S}_{1}}^{\text {cl. }}(t)=\frac{N_{\mathrm{S}_{1}}(t)}{N_{t r a j}}
$$

We refer to this estimate of the electronic population as "classical" and we indicate it as (cl.) in Fig. 6. In Fig. 6 we report as well TSH results (black) using the energy decoherence correction (with parameter 0.1 Ha), indicated as TSH-ED; only the classical estimate of the electronic population obtained from TSH-ED is shown in the figure.

Figure 6 reports the probability of the trans configuration as function of time as well (right panel). Exact results (red) are determined by integrating at all times the total nuclear density between $\varphi=-\frac{\pi}{2}$ and $\varphi=+\frac{\pi}{2}$ (and over all $q$ ), thus obtaining $P_{\text {cis }}(t)$, and then by defining $P_{\text {trans }}(t)=1-P_{\text {cis }}(t)$. Trajectory-based results are determined by counting at each time how many trajectories have a value of $\left|\varphi^{\alpha}(t)\right|>\frac{\pi}{2}$, independently of $q^{\alpha}(t)$, and by dividing this number by the total number of trajectories. CT-MQC results are shown in blue, CT-TSH in orange, TSH-ED in black, quantum trajectories (evolved with the TDVP) in lilac in Fig. 6 (right panel). Note that, when the trajectories are evolved with the TDVP, only their distribution in configuration and momentum space is available. For this reason, we can only compute the probability of the trans configuration using quantum trajectories and we do not have access to the electronic population.

Quantum (exact) results from Fig. 6 show that after around $80 \mathrm{fs}$ of dynamics in the excited state, the photoexcited wavepacket reaches the CIs and starts transferring population to the ground state. At the same time, the trans configuration is being formed as the wavepacket 

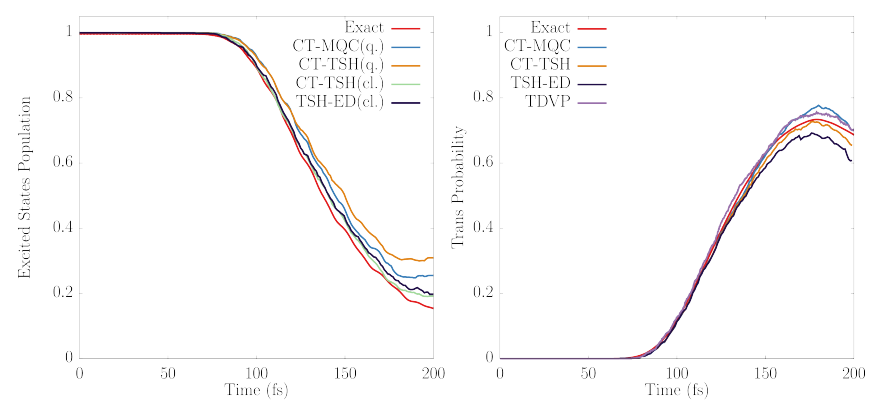

FIG. 6. Left: Population of the electronic excited state $\mathrm{S}_{1}$ as function of time from quantum dynamics (Exact, in red), CT-MQC (in blue), CT-TSH (in orange and in green), and TSH-ED (in black). For CT-MQC only the quantum (q.) estimate of the population is accessible, whereas for CT-TSH we compare the quantum (q.) and the classical (cl.) expressions, as defined in the text. For TSH-ED, only the classical (cl.) estimate is shown. Right: Probability of finding the system in the trans configuration as function of time from quantum dynamics (Exact, in red), CT-MQC (in blue), CT-TSH (in orange), TSH-ED (in black), and quantum trajectories (in lilac).

tends towards values of the reactive coordinates $|\varphi|>\frac{\pi}{2}$. While the $\mathrm{S}_{1}$ population keeps decreasing until $200 \mathrm{fs}$, i.e., the end of the simulated dynamics, the trans configuration reaches a maximum at around $180 \mathrm{fs}$ and decreases afterwards. This behavior suggests transfer of density from the trans basins to the cis basin on the $\mathrm{S}_{0}$ PES without population transfer, since the population of $\mathrm{S}_{1}$ does not increase after $180 \mathrm{fs}$. An additional feature that we can observe in the plot of the population of $\mathrm{S}_{1}$ is that the curve hints towards a stabilization after $200 \mathrm{fs}$.

The formation of the trans conformer is reproduced by the quantum trajectories (lilac) in extremely good agreement with exact results, indicating that a trajectorybased representation of nuclear dynamics for this retinal model is adequate to study the trans photo-product formation after excitation by light. However, TDVP results slightly deviate from the reference towards the end of the simulated dynamics, due to numerical errors in the time integration of Eq. (19) when the TDVP develops a highly oscillatory behavior (see Fig. 3 at time $100 \mathrm{fs}$ ).

CT-MQC results (blue) reproduce qualitatively and quantitatively well the expected behavior of the $\mathrm{S}_{1}$ population, eve though it seems that the time trace of the population reaches a plateau at around 180-190 fs. The trans probability as function of time is also reproduced extremely well, with the maximum only slightly overestimated if compared to the reference.

Interestingly, the quantum (orange) and classical (green) estimates of the electronic population in CT-TSH are very close all along the dynamics, even though this has not been imposed in the derivation of the algorithm. The quantum estimate is, however, closer to CT-MQC, as the population reaches a plateau at around 180-190 fs, which is not observed in the classical estimate. The classical estimate of the $S_{1}$ population resembles closely the reference curve as well as TSH-ED results. In addition, the prediction of the trans probability by CT-TSH is very close to the exact curve, and shows a slight (quantitative) improvement with respect to both CT-MQC and TSHED.

\section{CONCLUSIONS}

In this paper we reported the analysis of the relaxation dynamics through a conical intersection in a twodimensional retinal model and fully based on the exact factorization. The aim of the work is to validate approximate trajectory-based results obtained by applying CTMQC and CT-TSH against quantum wavepacket dynamics and against quantum trajectories, which represent for us the best trajectory-based results accessible within the exact factorization. To propagate quantum trajectories, we calculated numerically the time-dependent vector potential and time-dependent potential energy surface from the solution of the coupled electron-nuclear problem.

The agreement between quantum trajectoires and quantum wavepacket dynamics is excellent all along the simulated dynamics, even though the time-dependent potentials show complex interferences at long times due to the reduced dimensionality of the model. Despite this complex dynamical features, the approximate trajectorybased schemes CT-MQC and CT-TSH are in close agreement to quantum results, i.e., trajectories and wavepackets. Furthermore, CT-TSH shows a slightly improved quantitative agreement with the reference over CT-MQC.

\section{ACKNOWLEDGEMENTS}

This work was supported by the ANR Q-DeLight project, Grant No. ANR-20-CE29-0014 and by the ANR HYTRAJ project, Grant No. ANR-19-CE30-0039 of the French Agence Nationale de la Recherche.
[1] L. González and R. Lindh, Quantum Chemistry and Dynamics of Excited States: Methods and Applications (John Wiley \& Sons, Chichester, 2020).

[2] R. Crespo-Otero and M. Barbatti, Chem. Rev. 118, 7026 (2018).
[3] B. F. E. Curchod and T. J. Martínez, Chem. Rev. 118, 3305 (2018).

[4] F. Agostini and E. K. U. Gross, Eur. Phys. J. B 94, 179 (2021).

[5] M. Bonfanti, G. A. Worth, and I. Burghardt, in Quantum Chemistry and Dynamics of Excited States: Methods 
and Applications, edited by L. González and R. Lindh (John Wiley \& Sons, 2021).

[6] F. Agostini and B. F. E. Curchod, WIREs Comput. Mol. Sci. 9, e1417 (2019).

[7] B. Lasorne, G. A. Worth, and M. A. Robb, WIREs Comput. Mol. Sci. 1, 460 (2011).

[8] L. M. Ibele and B. F. E. Curchod, Phys. Chem. Chem. Phys. 22, 15062 (2020).

[9] M. Barbatti, WIREs Comput. Mol. Sci. 1, 620 (2011).

[10] S. Mai, P. Marquetand, and L. González, WIREs Comput. Mol. Sci. 8, e1370 (2018).

[11] H.-D. Meyer, WIREs Comput. Mol. Sci. 2, 351 (2012).

[12] J. Westermayr and P. Marquetand, Chem. Rev. 121, 9873 (2021).

[13] G. A. Worth and B. Lasorne, in Quantum Chemistry and Dynamics of Excited States: Methods and Applications, edited by L. González and R. Lindh (John Wiley \& Sons, 2021).

[14] G. W. Richings, I. Polyak, K. E. Spinlove, G. A. Worth, I. Burghardt, and B. Lasorne, Int. Rev. Phys. Chem. 34, 269 (2015).

[15] M. Ben-Nun and T. J. Martínez, J. Chem. Phys. 108, 7244 (1998).

[16] B. F. E. Curchod, in Quantum Chemistry and Dynamics of Excited States: Methods and Applications, edited by L. González and R. Lindh (John Wiley \& Sons, 2021).

[17] D. V. Makhov, W. J. Glover, T. J. Martinez, and D. V. Shalashilin, J. Chem. Phys. 141, 054110 (2014).

[18] V. M. Freixas, A. J. White, T. Nelson, H. Song, D. V. Makhov, D. Shalashilin, S. Fernandez-Alberti, and S. Tretiak, J. Phys. Chem. Lett. 12, 2970 (2021).

[19] M. Vacher, M. J. Bearpark, and M. A. Robb, Theor. Chem. Acc. 135, 187 (2016).

[20] A. Kirrander and M. Vacher, in Quantum Chemistry and Dynamics of Excited States: Methods and Applications, edited by L. González and R. Lindh (John Wiley \& Sons, 2021).

[21] J. C. Tully, J. Chem. Phys. 93, 1061 (1990).

[22] S. K. Min, F. Agostini, and E. K. U. Gross, Phys. Rev. Lett. 115, 073001 (2015).

[23] F. Agostini, S. K. Min, A. Abedi, and E. K. U. Gross, J. Chem. Theory Comput. 12, 2127 (2016).

[24] S. K. Min, F. Agostini, I. Tavernelli, and E. K. U. Gross, J. Phys. Chem. Lett. 8, 3048 (2017).

[25] C. Pieroni and F. Agostini, J. Chem. Theory Comput. 17, 5969 (2021).

[26] F. Agostini and E. K. U. Gross, in Quantum chemistry and dynamics of excited states: Methods and applications, edited by L. González and R. Lindh (Wiley, 2020) pp. 531-562.

[27] J.-K. Ha, I. S. Lee, and S. K. Min, J. Phys. Chem. Lett. 9, 1097 (2018).

[28] A. Abedi, N. T. Maitra, and E. K. U. Gross, Phys. Rev. Lett. 105, 123002 (2010).

[29] A. Abedi, N. T. Maitra, and E. K. U. Gross, J. Chem. Phys. 137, 22A530 (2012).

[30] B. F. E. Curchod and F. Agostini, J. Phys. Chem. Lett. 8, 831 (2017).

[31] F. Agostini and B. F. E. Curchod, Euro. Phys. J. B 91, 141 (2018).

[32] L. M. Ibele, B. F. E. Curchod, and F. Agostini, J. Phys. Chem. A 10.1021/acs.jpca.1c09604 (2022).

[33] A. Abedi, F. Agostini, Y. Suzuki, and E. K. U. Gross, Phys. Rev. Lett 110, 263001 (2013).
[34] L. Lacombe, N. M. Hoffmann, and N. T. Maitra, Phys. Rev. Lett. 123, 083201 (2019).

[35] E. Khosravi, A. Abedi, and N. T. Maitra, Phys. Rev. Lett. 115, 263002 (2015).

[36] F. Agostini, A. Abedi, Y. Suzuki, and E. K. U. Gross, Mol. Phys. 111, 3625 (2013).

[37] F. Agostini, A. Abedi, Y. Suzuki, S. K. Min, N. T. Maitra, and E. K. U. Gross, J. Chem. Phys. 142, 084303 (2015).

[38] Y. Suzuki, A. Abedi, N. T. Maitra, and E. K. U. Gross, Phys. Chem. Chem. Phys. 17, 29271 (2015).

[39] Y. Suzuki and K. Watanabe, Phys. Rev. A 94, 032517 (2016).

[40] F. Agostini, S. K. Min, and E. K. U. Gross, Ann. Phys. 527, 546 (2015).

[41] A. Scherrer, F. Agostini, D. Sebastiani, E. K. U. Gross, and R. Vuilleumier, Phys. Rev. X 7, 031035 (2017).

[42] M. Filatov, S. K. Min, and K. S. Kim, Mol. Phys. 117, 1128 (2019).

[43] M. Filatov, S. K. Min, and C. H. Choi, Phys. Chem. Chem. Phys. 21, 2489 (2019).

[44] I. S. Lee, J. Ha, D. Han, T. I. Kim, S. W. Moon, and S. K. Min, J. Comput. Chem. 42, 1755 (2021).

[45] P. Vindel-Zandbergen, S. Matsika, and N. T. Maitra, J. Phys. Chem. Lett. 13, 1785 (2022).

[46] P. Vindel-Zandbergen, L. M. Ibele, J.-K. Ha, S. K. Min, B. F. E. Curchod, and N. T. Maitra, J. Chem. Theory Comput. 17, 3852 (2021).

[47] S. Hahn and G. Stock, J. Phys. Chem. B 104, 1146 (2000).

[48] C. Pieroni, E. Marsili, D. Lauvergnat, and F. Agostini, J. Chem. Phys. 154, 034104 (2021).

[49] F. Talotta, F. Agostini, and G. Ciccotti, J. Phys. Chem. A 124, 6764 (2020).

[50] F. Agostini, I. Tavernelli, and G. Ciccotti, Euro. Phys. J. B 91, 139 (2018).

[51] F. Talotta, S. Morisset, N. Rougeau, D. Lauvergnat, and F. Agostini, J. Chem Theory Comput. 16, 4833 (2020).

[52] F. Agostini, B. F. E. Curchod, R. Vuilleumier, I. Tavernelli, and E. K. U. Gross, in Handbook of Materials Modeling, edited by W. Andreoni and S. Yip (Springer Netherlands, 2018) pp. 1-47.

[53] G. Gossel, F. Agostini, and N. T. Maitra, J. Chem. Theory Comput. 14, 4513 (2018).

[54] F. Agostini, Euro. Phys. J. B 91, 143 (2018).

[55] P. R. Holland, The Quantum Theory of Motion - An Account of the de Broglie-Bohm Causal Interpretation of Quantum Mechanics (Cambridge University Press, 1993).

[56] S. Hahn and G. Stock, Chem. Phys. 259, 297 (2000).

[57] L. Seidner and W. Domcke, Chem. Phys. 186, 27 (1994).

[58] T. V. Tscherbul and P. Brumer, Phys. Chem. Chem. Phys. 17, 30904 (2015).

[59] M. H. Farag, T. L. C. Jansen, and J. Knoester, J. Phys. Chem. Lett. 7, 3328 (2016).

[60] D. Dey and N. E. Henriksen, J. Phys. Chem. Lett. 11, $8470(2020)$.

[61] B. Balzer, S. Dilthey, S. Hahn, M. Thoss, and G. Stock, J. Chem. Phys. 119, 4204 (2003).

[62] P. E. Videla, A. Markmann, and V. S. Batista, J. Chem. Theory Comput. 14, 1198 (2018).

[63] B. Balzer and G. Stock, J. Phys. Chem. A 108, 6464 (2004). 
[64] M. Ndong, L. Bomble, D. Sugny, Y. Justum, and M. Desouter-Lecomte, Phys. Rev. A 76, 043424 (2007).

[65] I. Uspenskiy, B. Strodel, and G. Stock, Chem. Phys. 329, 109 (2006).

[66] B. Balzer, S. Hahn, and G. Stock, Chem. Phys. Lett. 379, 351 (2003).

[67] S. Hahn and G. Stock, J. Chem. Phys. 116, 1085 (2002).

[68] D. Lauvergnat, "ElVibRot-TnumTana Quantum Dynamics Code," https://github.com/lauvergn/ ElVibRot-TnumTan.

[69] H. Tal-Ezer and R. R. Kosloff, J. Chem. Phys. 81, 3967 (1984).
[70] H. Bessone, L.-M. Ibele, E. Marsili, F. Talotta, D. Lauvergnat, B. F. E. Curchod, and F. Agostini, "EFAC," (2021), EFAC.

[71] R. P. Feynman, Phys. Rev. 56, 340 (1939).

[72] D. Lauvergnat, "https://github.com/lauvergn/ QuantumModelLib/tree/OOP_branch," (2018), QuantumModelLib.

[73] F. Agostini, E. Marsili, and F. Talotta, "G-CTMQC," (2021), gitlab.com/agostini.work/g-ctmqc. 